\title{
Modifications of abundances of some bacteria are associated with the severity of IgA nephropathy in Chinese population
}

\section{ZhengXia Zhong}

Division of Nephrology, Department of Medicine, West China Hospital, Sichuan University, Chengdu, Sichuan, China

JiaXing Tan

Division of Nephrology, Department of Medicine, West China Hospital, Sichuan University, Chengdu,

Sichuan, China

Li Tan

Division of Nephrology, Department of Medicine, West China Hospital, Sichuan University, Chengdu, Sichuan, China

\section{Yi Tang}

Division of Nephrology, Department of Medicine, West China Hospital, Sichuan University, Chengdu, Sichuan, China

\section{ZhiCheng Qiu}

Division of Nephrology, Department of Medicine, Affiliated Hospital of Zunyi Medical University, Guizhou, China

\section{GaiQin Pei}

Division of Nephrology, Department of Medicine, West China Hospital, Sichuan University, Chengdu,

Sichuan, China

Wei Qin ( $\square$ ddqstrike@163.com )

Sichuan University West China Hospital https://orcid.org/0000-0003-4246-5431

\section{Research}

Keywords: IgA nephropathy, Modifications of abundances of bacteria, 16S ribosomal RNA, blood biomarkers, severity

Posted Date: July 9th, 2020

DOI: https://doi.org/10.21203/rs.3.rs-40704/v1

License: (c) (i) This work is licensed under a Creative Commons Attribution 4.0 International License. Read Full License 


\section{Abstract \\ Background}

Immunoglobulin A nephropathy (IgAN) is a common autoimmune glomerular disease, the classic manifestations of which are hematuria and proteinuria. The pathogenesis of IgAN has been associated with dysregulated intestinal mucosal immunity. However, whether gut microbial modifications play a part in IgAN remains unclear.

\section{Methods}

Blood and fecal samples were collected from $52 \mathrm{IgAN}$ patients and 25 healthy controls (HCs). The gut microbiome was analyzed by $16 \mathrm{~S}$ ribosomal RNA (rRNA) gene sequencing, followed by analyses of gut microbiota composition. Furthermore, the levels of galactose-deficient IgA1 (Gd-lgA1), soluble cluster of differentiation 14 (SCD14), lipopolysaccharide binding protein (LBP), intercellular adhesion molecule-1 (ICAM-1), and C-reactive protein (CRP) was quantified by enzyme-linked immunosorbent assay (ELISA).

\section{Results}

Substantial differences in gut microbiota were found between IgAN patients and HCs $(P<0.05)$. The abundance of pathogenic bacteria (Bacteroides and Escherichia-Shigella) was significantly higher in IgAN patients than in $\mathrm{HCs}$, while that of beneficial strains (Bifidobacterium and Blautia spp.) was lower. Higher proportions of Escherichia-Shigella and lower proportions of Bifidobacterium spp. were observed in IgAN patients with a high urine RBC count ( $\geq 10 / \mathrm{HP}$ ) and proteinuria ( $\geq 1 \mathrm{~g} / 24 \mathrm{~h}$ ) levers. Spearman's correlation analysis was used to assess the association between gut microbiota and biomarkers in IgAN patients. The results showed that the genus Prevotella 7 was negatively correlated with Gd-IgA1, LBP, sCD14, and ICAM-1, while Bifidobacterium spp. presented a significant inverse relationship with LBP and Gd-IgA1. Additionally, Escherichia-Shigella was negatively correlated with Prevotella 7.

\section{Conclusions}

In IgAN patients, gut modifications were characterized by an increase in the numbers of pathogenic bacteria and a reduction in the levers of beneficial bacteria. Our results suggest that disturbance of the intestinal microflora might play a critical part in the severity of $\operatorname{lgAN}$, and may be a potential therapeutic target.

\section{Introduction}

Immunoglobulin A nephropathy (IgAN), characterized by diffused IgA deposition in the glomerular mesangium, is a leading cause of end-stage renal disease (ESRD) in adults [1]. Long-term research has 
indicated that approximately $20-40 \%$ of IgAN patients progress to ESRD within 20-40 years, making it an important public health problem [2,3]. However, the pathogenesis of IgAN remains unclear.

Recently, modifications of the gut microbiota have been recognized as a risk factor for several kidney diseases, including diabetic nephropathy, chronic kidney diseases, cardiovascular disease, inflammatory bowel disease, obesity, and several cancers [3-5]. Although a previous study has reported that the gut microbial community in IgAN patients is substantially changed when compared to healthy controls [6], the precise differences and the relationship between the microbes and severity of IgAN clinical manifestations are poorly understood. Therefore, we analyzed the fecal gut microbiota of IgAN patients in Chinese population to evaluate the potential correlation between the compositions of the gut microbiome and disease severity.

\section{Materials And Methods}

\section{Study Subjects}

A total of 52 patients with IgAN diagnosis confirmed by biopsy and 25 healthy controls (HCs) subjects were enrolled between June 2018 and July 2019 from West China Hospital of Sichuan University. The diagnostic criteria for IgAN were based on the KDIGO Clinical Practice Guidelines for Glomerulonephritis [7]. Patients with the same diet and lifestyle habits were included in this study. Individuals with secondary IgAN, autoimmune disorders, diabetes mellitus, acute myocardial infarction, stroke, abnormal liver function, malignancies, digestive tract disease, and those who had received antibiotic treatment within 4 weeks were excluded. This study was performed in accordance with the Helsinki Declaration and approved by the Biomedical Ethics Committee of West China Hospital of Sichuan University. Written informed consent was acquired from all participants.

\section{Baseline Data and Laboratory Measurements}

Age, gender, systolic blood pressure, diastolic blood pressure, serum creatinine, estimated glomerular filtration rate (eGFR) calculated by the Chronic Kidney Disease Epidemiology Collaboration (CKD-EPI) equation, blood urea nitrogen (BUN), urine protein, and hematuria were recorded for each study subject [8].

A fasting venous blood sample was obtained from all the participants. Serum was separated by centrifugation $\left(3,000 \square \mathrm{g}\right.$ at $4{ }^{\circ} \mathrm{C}$ for $10 \mathrm{~min}$ ) and immediately frozen at $-80^{\circ} \mathrm{C}$ for further use. Fresh fecal samples of each subject were collected into a sterile container and immediately stored at $-80^{\circ} \mathrm{C}$.

Serum galactose-deficient IgA1 (Gd-lgA1, U/mL), lipopolysaccharide (LPS) binding protein (LBP, $\mu \mathrm{mol} / \mathrm{L}$ ), soluble cluster of differentiation 14 (sCD14, $\mathrm{ng} / \mathrm{mL}$ ), intercellular adhesion molecule-1 (ICAM-1, ng/mL), and C-reactive protein (CRP, $\mathrm{mg} / \mathrm{L}$ ) were detected by enzyme-linked immunosorbent assay (ELISA) (Shanghai Enzyme-linked Biotechnology Co., Ltd, China). Repeated measurements were performed based on the manufacturer's protocol [9]. 


\section{Sequencing of the $16 \mathrm{~S}$ rRNA Gene}

DNA extraction from fecal microbiota was performed using the E.Z.N.A.® Soil DNA Kit (Omega Bio-tek, Norcross, GA, USA). The amount and purity of microbial DNA were verified by a NanoDrop 2000 UV-vis spectrophotometer (Thermo Scientific, Wilmington, DE, USA). Agarose gel electrophoresis (1\%) was used to evaluate the integrity and the size of the final DNA.

The universal primer pair 338F (5'-ACTCCTACGGGAGGCAGCAG-3') and 806R (5'GGACTACHVGGGTWTCTAAT-3') was used to PCR-amplify the 16S ribosomal RNA (rRNA) V3-V4 hypervariable regions using a GeneAmp 9700 thermocycler system ( $\mathrm{ABI}, \mathrm{USA}$ ). The reaction conditions comprised denaturation at $95^{\circ} \mathrm{C}$ for $3 \mathrm{~min}, 27$ cycles of $95^{\circ} \mathrm{C}$ for $30 \mathrm{~s}$, annealing at $55^{\circ} \mathrm{C}$ for $30 \mathrm{~s}$, extension at $72{ }^{\circ} \mathrm{C}$ for $45 \mathrm{~s}$, and a final a extension step at $72{ }^{\circ} \mathrm{C}$ for 10 min. Reaction mixtures $(20 \mu \mathrm{L})$ included FastPfu Buffer, $2.5 \mathrm{mM}$ dNTPs, $5 \mu \mathrm{M}$ of each forward and reverse primer, FastPfu Polymerase, and template DNA. The PCR products were extracted from $2 \%$ agarose gels using the AxyPrep DNA Gel Extraction Kit (Axygen Biosciences, Union City, CA, USA) and quantified using QuantiFluor ${ }^{\text {TM }}$-ST (Promega, USA) [10].

The purified amplicons were pooled equimolarly and paired-end sequenced on the lllumina MiSeq platform (Illumina, San Diego, CA, USA). The raw sequencing reads were recorded in the fastq format and quality-filtered by Trimmomatic. FLASH was used to merge all the raw sequencing reads. Reads were clustered into operational taxonomic units (OTUs) at a 97\% similarity cutoff using UPARSE (version 7.1; http://drivve5.com/uparse), where a new greedy algorithm was adopted that simultaneously performed chimera filtering and OTU clustering. The ribosomal database project (RDP) classifier algorithm (http://rdp.cme.msu.edu/) against the Silva (SSU123) 16S rRNA database was used with a 70\% confidence threshold to further categorize each 16S rRNA sequence.

\section{Statistical Analysis}

For baseline data and laboratory findings, quantitative data were presented either as medians with interquartile range or means \pm standard deviation (SD), whereas variables were presented as percentages and compared by the Student's t-test, Wilcoxon rank-sum test, chi-square test, or Fisher's exact test. Statistical analyses were performed with SPSS version 22.0 (IBM SPSS, Chicago, IL, USA).

Data for the 16S rRNA were processed on the Majorbio Cloud Platform (www.majorbio.com). All the data were analyzed at the genus level. Permutational multivariate analysis of variance (PERMANOVA) was carried out using the R package "vegan". The permuted P-value was acquired from 10,000 permutations. Sample size and sequence accuracy were determined by pan/core genome species analysis and rarefaction curves. Alpha diversity, including Ace, Chao, Coverage, Shannon, Simpson, and Sobs diversity indices, and beta diversity, including principal coordinate analysis (PCoA) and Venn diagrams, were calculated to determine community richness and diversity. Taxonomic differences from the phylum to genus level in IgAN and HCs were directly determined by the linear discriminant analysis (LDA) effect size (LEfSe) algorithm, where an LDA score $>2.0$ was considered to be significant. Differential abundance at 
the phylum and genus levels was detected by the Wilcoxon rank-sum test. Connections between demographic data/biochemical indicators and bacterial genera were calculated by Spearman's rank correlation coefficient. Two-tailed P-values were generated for all parameters and P-values $<0.05$ were considered significant.

\section{Results}

\section{Baseline Characteristics of the Study Subjects}

In this cross-sectional study, 52 IgAN patients and $25 \mathrm{HCs}$ were enrolled according to the specified inclusion and exclusion criteria (see Materials and Methods). The basic information for IgAN patients and HCs is shown in Table 1. Systolic blood pressure, diastolic blood pressure, and serum levels of creatinine, e-GFR, BUN, urine protein, and red blood cell numbers in urine were significantly higher in IgAN patients than in $\mathrm{HCs}(\mathrm{P}<0.05)$.

\section{Composition of Gut Microbiota}

Analysis of Pan and Core genomes and rarefaction curves at the genus level indicated that the data in our study were large enough and valid (Fig. 1). We evaluated alpha and beta diversity to compare community richness and diversity between the gut microbiota of IgAN patients and HCs. Although alpha diversity analysis showed no significant differences in taxon richness and evenness, there was a clear separation in the composition of gut microbiota between the IgAN patients and HCs (Ace, $\mathrm{P}=0.073$; Chao, $P=0.077$; Sobs, $P=0.088$ ) (Table 2 ). These results suggested that bacterial community diversity was altered between the two groups.

Beta diversity analysis also demonstrated that the overall structure of the intestinal microbiomes differed significantly between IgAN patients and HCs $(P=0.005$; Fig. $2 A)$, which was further confirmed by Venn diagram analysis (Fig. 2a). Overall, 437 genera were detected at a $97 \%$ sequence homology cutoff for the 16S rRNA gene. Of these genera, 287 were shared between IgAN patients and HCs, 18 were specific for the $\mathrm{HCs}$, and 132 were unique to IgAN patients. Overall, the gut microbiome composition of IgAN patients differed from that of HCs.

\section{Specific Differences from Phylum to Genus Levels in IgAN Patients and HCs}

To further explore the differences in microflora between IgAN patients and HCs, we used the LEfSe algorithm. The results indicated that 3 phyla, 3 classes, 3 orders, 5 families, and 12 genera were enriched in IgAN patients, while 2 phyla, 2 classes, 5 orders, 7 families, and 28 genera were enriched in HCs (LDA score $>2.0, \mathrm{P}<0.05$ ) (Fig. 3a). At the phylum level, the relative abundance of Bacteroidetes was higher in IgAN patients while that of Firmicutes was higher in HCs. At the genus level, the relative abundance of Bacteroides was higher in IgAN patients, whereas that of Subdoligranulum was higher in HCs. A cladogram was then generated to directly visualize and compare the phylogenetic distribution from the 
phylum to genus level between the two groups, with the results demonstrating that significant differences existed at each taxonomic level analyzed $(P<0.05$; Fig. 3b).

At the phylum level, a Wilcoxon rank-sum test indicated that the proportions of Bacteroidetes and Fusobacteria were markedly higher in IgAN patients than in $\mathrm{HCs}(\mathrm{P}<0.05 ; \mathrm{Fig}$. 4a). Additionally, IgAN patients had a higher proportion of Proteobacteria, although the difference was not significant $(\mathrm{P}=$ 0.066). Conversely, the proportions of Firmicutes, Actinobacteria, and Tenericutes were significantly higher in the fecal microbiota of HCs $(P<0.05)$. A genus-level comparison between HCs and IgAN patients demonstrated that the abundances of Bacteroides, Escherichia-Shigella, and Lachnoclostridium were significantly increased in IgAN patients when compared with those of $\mathrm{HCs}(\mathrm{P}<0.05$; Fig. 4b). However, IgAN patients also presented a significant reduction in the proportions of Blautia, Subdoligranulum, Prevotella 9, genus_Eubacterium hallii, and Bifidobacterium $(\mathrm{P}<0.05)$. The community bar plot analysis also showed differences in composition at the genus level (Fig. 4c), although the differences were not significant.

\section{Relationship between Gut Microbiota and Clinical Characteristics}

IgAN patients were divided into subgroups based on the levels of proteinuria and hematuria to evaluate whether intestinal microbes were related to the severity of IgAN. As most patients enrolled in our study were at early stages of chronic kidney disease (CKD 1 and CKD 2 stages), subgroup analysis based on the eGFR level was discontinued. As shown in Table 3, patients with a higher urine RBC count $(\geq 10 / H P)$ count presented a higher abundance of Escherichia-Shigella $(P=0.016)$ and a lower abundance of Bifidobacterium spp. $(P=0.055)$ than patients with a urine RBC count $<10 / H P$. Patients with different levels of urine protein had significantly different abundances of Subdoligranulum spp. $(P<0.05)$. Moreover, the abundance of Escherichia-Shigella was marginally higher, while that of members of the Bifidobacterium genus was lower $(P=0.06)$, in patients with proteinuria $\geq 1 \mathrm{~g} / 24 \mathrm{~h}(P=0.075)$.

Because immune reactions and inflammation have a role in IgAN pathogenesis, serum concentrations of immune and inflammatory factors, such as Gd-IgA1, LBP, sCD14, ICAM-1, and CRP, were measured along with routine laboratory indexes. We found that serum levels of Gd-IgA1, LBP, SCD14, and ICAM-1, and CRP levels were significantly increased in IgAN patients when compared with those of HCs (Fig. 5).

Spearman's correlation analysis was performed only in the IgAN group to investigate the relationship between bacteria and inflammation indexes in the disease state. The genus Prevotella 7 was negatively correlated with Gd-IgA1, LBP, sCD14, and ICAM-1 $(r=-0.356, P=0.014 ; r=-0.322, P=0.014 ; r=-0.321, P$ $=0.028 ; r=-0.311, P=0.033$, respectively). Meanwhile, Bifidobacterium spp. had a marked inverse relationship with LBP and Gd-IgA1 $(r=-0.301, P=0.040 ; r=-0.266, P=0.070$, respectively). Additionally, Escherichia-Shigella was negatively correlated with Prevotella $7 \mathrm{spp} .(r=-0.305, \mathrm{P}=0.037)$, indicating that there was also interaction among microbiota, as shown in Table 4. Combined with previous results, it was reasonable to draw a conclusion that the gut microbiota might be associated with inflammatory state in IgAN. 


\section{Discussion}

This was a preliminary cross-sectional cohort study, the investigation of fecal microbiota diversity and abundance of Chinese IgAN patients was performed by high-throughput sequencing and QPCR analyses. IgAN is the most prevalent primary glomerulonephritis in China [11]. Immune reaction, bacterial infection and inflammation have been reported as etiological factors of $\lg A N$. More than one hundred trillion microbes exist in the intestinal tract, one of the biggest immune organs of the human body [12]. An increasing number of studies have revealed that the gut-kidney axis may contribute to the pathogenesis of numerous diseases $[4,5]$. However, only one pilot study with a relatively small number of samples has reported the dysregulation of the gut microbiome in IgAN patients [6]. Therefore, we undertook this study to explore whether the gut microbiota is associated with the manifestations and severity of IgAN.

The 16S rRNA sequencing results showed that the composition of the gut microbiome from the phylum to genus levels differed between IgAN patients and HCs. Cladogram and LEfSe analysis suggested that the relative abundances of the dominant bacterial communities were different. Subdoligranulum was the most abundant genus in $\mathrm{HCs}$, while Bacteroides was dominant in IgAN patients, indicating that the composition of intestinal flora was changed in IgAN patients. Moreover, marked differences in the percentages of specific bacteria were also noted, especially the overrepresentation of Bacteroides, Escherichia-Shigella, and Lachnoclostridium, and the underrepresentation of Blautia, Subdoligranulum, Prevotella 9, genus_Eubacterium hallii, and Bifidobacterium in IgAN patients. Bifidobacterium spp. are one of the most common probiotics in healthy people, and play an essential role in the gastrointestinal tract, including intestinal biological barrier function and immune enhancement [13], while Eubacterium hallii, Blautia spp., and Prevotella spp. are also beneficial for human physiology [14, 15]. Therefore, decreased levels of these beneficial bacteria may compromise the immune regulatory function in the intestine. Bacteroides and Escherichia-Shigella are considered pathogenic bacteria that can cause intestinal infections under conditions of lower resistance $[10,16,17]$. An increase in the numbers of these bacteria may lead to local infection and activation of immune responses, which may lead to increased IgA synthesis. It is noteworthy that the physiological function of the bacteria shown above is not fully understood. But limited data suggests that $g$-Eubacterium hallii, g-Blautia and $g$-Prevotella are beneficial for human physiology. Accordingly, we speculated that gut microbial dysbiosis, characterized by increase in pathogenic bacteria and a decrease in beneficial bacteria, were relevant to IgAN.

The results of the current study also indicated that a strong correlation existed between intestinal microflora and the clinical characteristics of IgAN patients. Patients with higher urine RBC counts $(\geq$ $10 / \mathrm{HP}$ ) or proteinuria levels ( $\geq 1 \mathrm{~g} / 24 \mathrm{~h}$ ) had a higher percentage of Escherichia-Shigella and lower percentage of Bifidobacterium, suggesting that gut microbial modifications might be associated with the clinical severity of IgAN. Moreover, IgAN patients presenting with proteinuria levels $\geq 1 \mathrm{~g} / 24 \mathrm{~h}$ were substantially more likely to progress to end-stage renal disease, indicating that intestinal flora imbalance, characterized by an increased abundance of pathogenic bacteria and reduction in that of beneficial strains, may be relevant to IgAN progression $[18,19]$. Recently, the substantial diversity in bacterial composition from tonsillar crypts of IgA patients was detected by Watanabe $\mathrm{H}$ [20]. In their study, 
abundance of Prevotella spp., Haemophilus spp., Porphyromonas spp. and Treponema spp. was significantly higher in IgAN patients. But the changes in microbiota in tonsils were not the same as the changes in intestinal flora. De Angelis $\mathrm{M}$ et al. has proposed that some genera/species of Ruminococcaceae, Lachnospiraceae, Eubacteriaceae and Streptococcaeae were increased in patients with IgAN, but the abundance of Bifidobacteriaceae was markedly decreased [6]. Notably, both of us had found that the reduction of probiotic bacteria was associated with IgAN, suggesting that the modifications of gut microbiota did exist in IgA patients. However, changes in the abundance of other types of gut microbiota were not completely the same as ours, which might result from genetic diversities and differences in environment and eating habits. In addition, Chemouny et al. has reported that antibiotics, depleting gut microbiota efficiently, could ameliorate clinicopathological changes in humanized mice with IgAN [21]. As for the reason why we thought that the modification of gut microbiota was associated with the progression of IgAN, previous studies have reported that the higher levels of proteinuria and hematuria could serve as biomarkers of poor prognosis, which could guide the treatment $[22,23]$. Therefore, it was reasonable to speculate that modification of gut microbiota might be correlated with poor prognosis since we indicated that a significantly higher abundance of Escherichia-Shigella and a markedly lower abundance of Bifidobacterium spp. were observed in patients with higher levels of hematuria or proteinuria. Together, commensal bacteria might have effective effect on IgAN progression, but the exact mechanism remains unclear.

In our study, the serum concentrations of immune and inflammatory markers such as LBP, SCD14, ICAM1 , and CRP were also measured in IgAN patients. The results showed that the levels of all these markers were substantially higher in IgAN patients than in HCs. Following infection by gram-negative bacteria, LBP has a role in LPS aggregation, which leads to inflammation [24]. CD14, a glycolipid-anchored membrane glycoprotein, is generally regarded as a receptor for the LPS/LBP complex [25]. Both LBP and sCD14 are key molecules in the innate immune response [26, 27]. Patients with CKD have elevated levels of SCD14 and an increased risk of cardiovascular disease and death [28]. ICAM-1 can regulate immune reactions and may be associated with the pathogenesis of glomerulonephritis [29]. We also found that decreases in the levels of normal bacteria such as members of the genera Prevotella and Bifidobacterium were related to increased levels of Gd-IgA1, LBP, SCD14 and ICAM-1. Moreover, an increased abundance of the pathogenic Escherichia-Shigella was associated with decreased levels of normal bacteria (Prevotella 7). The pathogenic bacteria that showed increased abundances, such as Bacteroides and Escherichia-Shigella, were gram-negative, and these bacteria can release LPS and stimulate the secretion of LBP, sCD14, and other inflammatory markers. Based on our data, we presumed that gut microbiota might impact on renal impairment through inflammatory signaling pathways. Recently, depletion of gut microbiota using antibiotics was reported to ameliorate clinicopathological changes in a mouse model of $\lg \mathrm{AN}[21]$.

Several limitations must be taken into account in our study. First, as a cross-sectional study, we did not investigate the association between flora imbalance and prognosis. Second, environmental factors and food habits might influence the gut microbiome, and our results may only be applicable to Chinese IgAN patients. Third, this experiment did not initially design a comparison of the intestinal flora of IgAN and 
other glomerulonephritis. Finally, as majority of patients enrolled in our study were at the early stages of chronic kidney disease (CKD 1 and CKD 2 stages), the analysis based on the eGFR level was not processed. The relationships between gut microbiota and pathogenesis of IgAN are being carried out in further studies. Moreover, this was an observational study and no intervention was applied. Interventional studies using antibiotics or probiotics should be performed in the future.

\section{Conclusions}

Taken together, our results demonstrated that gut microbiota composition was significantly different between IgAN patients and HCs, and this difference was characterized as an increase in the abundance of opportunistic pathogenic bacteria and reduction in that of beneficial bacteria. Moreover, the gut microbiota was highly related to the clinical manifestations and inflammatory indicators of IgAN patients.

\section{Abbreviations}

IgAN: immunoglobulin A nephropathy; 16S rRNA:16S ribosomal RNA; Gd-IgA1:Galactose-deficient IgA1; SCD14:soluble cluster of differentiation 14, LBP:lipopolysaccharide binding protein; ICAM-1:intercellular adhesion molecule-1; CRP:C-reactive protein; KDIGO:kidney disease:improving global outcomes; CKD:chronic kidney disease.

\section{Declarations}

\section{Ethics approval and consent to participate}

This research was approved by the ethical committee of West China Hospital of Sichuan University.

\section{Competing interests}

The authors declare that the research was conducted in the absence of any commercial or financial relationships that could be construed as a potential conflict of interest.

\section{Availability of data and materials}

The datasets generated and/or analysed during the current study are not publicly available due to privacy policy but are available from the corresponding author on reasonable request.

\section{Consent for publication}

Written informed consents were signed by all the patients. 


\section{Funding}

This study received no external funding.

\section{Author Contributions}

QW designed the study and critically revised the work. ZZX and TJX performed the experiments $\square$ collected data and wrote the manuscript. ZZX, TJX, and TL performed the statistical analyses. TY, PGQ, and QZC collected the blood and fecal samples. All authors read and approved the final manuscript.

\section{Acknowledgements}

Thanks to all the volunteers and researchers who participated in this work.

\section{References}

1. Rodrigues JC, Haas M, Reich HN. IgA Nephropathy. Clin J Am Soc Nephrol. 2014;12(4):677-86.

2. Coppo R. Corticosteroids in IgA Nephropathy: Lessons from Recent Studies. J Am Soc Nephrol. 2017;28(1):25-33.

3. Cho I, Blaser MJ. The human microbiome: at the interface of health and disease. Nat Rev Genet. 2012;13(4):260-70.

4. Kikuchi K, Saigusa D, Kanemitsu Y, et al. Gut microbiome-derived phenyl sulfate contributes to albuminuria in diabetic kidney disease. Nat Commun. 2019;10(1):1835.

5. Li Y, Su X, Zhang L, et al. Dysbiosis of the gut microbiome is associated with CKD5 and correlated with clinical indices of the disease: a case-controlled study. J Transl Med. 2019;17(1):228.

6. De Angelis $M$, Montemurno $E$, Piccolo $M$, et al. Microbiota and metabolome associated with immunoglobulin A nephropathy (IgAN). PLoS One. 2014;9(6):e99006.

7. Radhakrishnan J, Cattran DC. The KDIGO practice guideline on glomerulonephritis: reading between the (guide)lines-application to the individual patient. Kidney Int. 2012;82(8):840-56.

8. Levey AS, Stevens LA, Schmid CH, et al. A new equation to estimate glomerular filtration rate. Ann Intern Med. 2009;150(9):604-12.

9. Tsukamoto H, Takeuchi S, Kubota K, et al. Lipopolysaccharide (LPS)-binding protein stimulates CD14-dependent Toll-like receptor 4 internalization and LPS-induced TBK1-IKKE-IRF3 axis activation. J Biol Chem. 2018;293(26):10186-201.

10. Tao S, Li L, Li L, et al. Understanding the gut-kidney axis among biopsy-proven diabetic nephropathy, type 2 diabetes mellitus and healthy controls: an analysis of the gut microbiota composition. Acta Diabetol. 2019;56(5):581-92. 
11. Bartosik LP, Lajoie G, Sugar L, et al. Predicting progression in IgA nephropathy. Am J Kidney Dis. 2001;38(4):728-35.

12. Ahluwalia B, Magnusson MK, Öhman L. Mucosal immune system of the gastrointestinal tract: maintaining balance between the good and the bad. Scand J Gastroenterol. 2017;52(11):1185-93.

13. Kruis W, Fric P, Pokrotnieks J, et al. Maintaining remission of ulcerative colitis with the probiotic Escherichia coli Nissle 1917 is as effective as with standard mesalazine. Gut. 2004;53(11):1617-23.

14. Hiippala $K$, Jouhten $H$, Ronkainen $A$, et al. The Potential of Gut Commensals in Reinforcing Intestinal Barrier Function and Alleviating Inflammation. Nutrients. 2018;10(8):988.

15. Ozato N, Saito S, Yamaguchi T, et al. Blautia genus associated with visceral fat accumulation in adults 20-76 years of age. NPJ Biofilms Microbiomes. 2019;5:28.

16. Zhu Y, He C, Li X, et al. Gut microbiota dysbiosis worsens the severity of acute pancreatitis in patients and mice. J Gastroenterol. 2018;54(4):347-58.

17. Chen YH, Bai J, Wu D, et al. Association between fecal microbiota and generalized anxiety disorder: Severity and early treatment response. J Affect Disord. 2019;259:56-66.

18. Reich HN, Troyanov S, Scholey JW, et al. Toronto Glomerulonephritis Registry. Remission of proteinuria improves prognosis in IgA nephropathy. J Am Soc Nephrol. 2007;18(12):3177-83.

19. Barbour SJ, Reich HN. Risk Stratification of Patients With IgA Nephropathy. Am J Kidney Dis. 2012;59(6):865-73.

20. Watanabe $\mathrm{H}$, Goto $\mathrm{S}$, Mori $\mathrm{H}$, et al. Comprehensive microbiome analysis of tonsillar crypts in IgA nephropathy. Nephrol Dial Transplant. 2017;32(12):2072-9.

21. Chemouny JM, Gleeson PJ, Abbad L, et al. Modulation of the microbiota by oral antibiotics treats immunoglobulin A nephropathy in humanized mice. Nephrol Dial Transplant. 2019;34(7):1135-44.

22. Yu GZ, Guo L, Dong JF, et al. Persistent Hematuria and Kidney Disease Progression in IgA Nephropathy: A Cohort Study. Am J Kidney Dis. 2020;76(1):90-9.

23. Reich HN, Troyanov S, Scholey JW, et al. Toronto Glomerulonephritis Registry. Remission of proteinuria improves prognosis in IgA nephropathy. J Am Soc Nephrol. 2007;18(12):3177-83.

24. Park BS, Lee JO. Recognition of lipopolysaccharide pattern by TLR4 complexes. Exp Mol Med. 2013;45(12):e66.

25. Jerala R. Structural biology of the LPS recognition. Int J Med Microbiol. 2007;297(5):353-63.

26. Wang SC, Klein RD, Wahl WL, et al. Tissue coexpression of LBP and CD14 mRNA in a mouse model of sepsis. J Surg Res. 1998;76(1):67-73.

27. Vangroenweghe $F$, Rainard P, Paape M, et al. Increase of Escherichia coli inoculum doses induces faster innate immune response in primiparous cows. J Dairy Sci. 2004;87(12):4132-44.

28. Poesen R, Ramezani A, Claes K, et al. Associations of Soluble CD14 and Endotoxin with Mortality, Cardiovascular Disease, and Progression of Kidney Disease among Patients with CKD. Clin J Am Soc Nephrol. 2015;10(9):1525-33. 
29. Chow J, Hartley RB, Jagger C, et al. ICAM-1 expression in renal disease. J Clin Pathol. 1992;45(10):880-4.

\section{Tables}

Table1: Baseline characteristics between IgAN patients and $\mathrm{HCs}$

\begin{tabular}{llll}
\hline Characteristics & IgAN & HCs & P value \\
Simple size & 52 & 25 & \\
\hline Age (years) & $35.0 \pm 9.1$ & $31.5 \pm 5.4$ & 0.08 \\
Male gender $(\%)$ & $24(46.2 \%)$ & $12(48.0 \%)$ & 0.88 \\
SBP $(\mathrm{mmHg})$ & $126.9 \pm 17.7$ & $111.6 \pm 6.2$ & $\mathrm{P}<0.001$ \\
$\mathrm{DBP}(\mathrm{mmHg})$ & $86.1 \pm 13.1$ & $69.4 \pm 3.2$ & $\mathrm{P}<0.001$ \\
$\mathrm{sCr}(\mathrm{umol} / \mathrm{L})$ & $82.5(70.0-108.1)$ & $61.0(55.5-70.0)$ & $\mathrm{P}<0.001$ \\
eGFR $\left(\mathrm{mL} / \mathrm{min} / 1.73 \mathrm{~m}^{2}\right)$ & $90.4(68.0-116.1)$ & $116.9(114.0-125.9)$ & $\mathrm{P}<0.001$ \\
BUN (umol/L) & $6.4 \pm 3.34$ & $4.8 \pm 0.8$ & 0.005 \\
Urine protein $(\mathrm{g} / 24 \mathrm{~h})$ & $1.85 \pm 2.1$ & $0.06 \pm 0.03$ & $\mathrm{P}<0.001$ \\
U-RBC/HPF & $15(0-282)$ & $0(0-2)$ & 0.024 \\
\hline
\end{tabular}

Abbreviations: IgAN, IgA nephropathy; HCs, heathy controls; SBP, systolic blood pressures; DBP, diastolic blood pressure; $\mathrm{Cr}$, serum creatinine; eGFR, estimated glomerular filtration rate; BUN, blood urea nitrogen; U-RBC/HPF, red blood cell per high power field in urine.

\section{Table2: $\alpha$-Diversity between IgAN patients and HCs}

\begin{tabular}{llll}
\hline $\begin{array}{l}\text { Estimators } \\
\text { Simple size }\end{array}$ & IgAN (mean \pm SD) & HCs $($ mean \pm SD $)$ & P value \\
\hline ace & $341.77 \pm 156.59$ & $350.48 \pm 74.89$ & \\
chao & $342.75 \pm 155.72$ & $356.50 \pm 74.22$ & 0.073 \\
coverage & $0.9985 \pm 0.0011$ & $0.9988 \pm 0.0003$ & 0.077 \\
shannon & $3.29 \pm 0.63$ & $3.23 \pm 0.75$ & 0.181 \\
simpson & $0.10 \pm 0.07$ & $0.14 \pm 0.15$ & 0.858 \\
sobs & $281.50 \pm 129.96$ & $296.12 \pm 67.94$ & 0.815 \\
\hline
\end{tabular}

Abbreviations: IgAN, IgA nephropathy; HCs, heathy controls; SD, standard deviation. 


\begin{tabular}{|c|c|c|c|c|c|c|}
\hline & \multicolumn{2}{|c|}{ Proteinuria } & \multirow{2}{*}{$P$ value } & \multicolumn{2}{|c|}{ Hematuria } & \multirow{2}{*}{$\mathrm{p}$ value } \\
\hline & $<1 \mathrm{~g} / 24 \mathrm{~h}$ & $\geq 1 \mathrm{~g} / 24 \mathrm{~h}$ & & $<10 / \mathrm{HP}$ & $\geq 10 / \mathrm{HP}$ & \\
\hline Numbers & 23 & 29 & & 21 & 31 & \\
\hline g-Bacteroides & $0.257(0.093-0.506)$ & $0.176(0.043-0.245)$ & 0.019 & $0.208 \pm 0.226$ & $0.226 \pm 0.172$ & 0.745 \\
\hline g-Blautia & $0.027 \pm 0.026$ & $0.049 \pm 0.053$ & 0.082 & $0.039 \pm 0.048$ & $0.041 \pm 0.043$ & 0.898 \\
\hline g-Subdoligranulu & $0.0130(0.0006-0.0280)$ & $0.0299(0.0025-0.0628)$ & 0.037 & $0.028 \pm 0.029$ & $0.032 \pm 0.043$ & 0.711 \\
\hline g-Prevotella-9 & $0.0000(0.0000-0.0000)$ & $0.0000(0.0000-0.0000)$ & 0.156 & $0.0008 \pm 0.0032$ & $0.0006 \pm 0.0030$ & 0.824 \\
\hline g-Prevotella-7 & $0.0025(0.0005-0.0107)$ & $0.0044(0.0017-0.0129)$ & 0.119 & $0.0124 \pm 0.0189$ & $0.009 \pm 0.0149$ & 0.491 \\
\hline $\mathrm{g}$-Bifidobacterium & $0.0024(0.0011-0.0136)$ & $0.0008(0.0001-0.0033)$ & 0.06 & $0.0020(0.0020-0.0180)$ & $0.0015(0.0003-0.0033)$ & 0.055 \\
\hline g-Escherichia-Shigella & $0.042 \pm 0.082$ & $0.049 \pm 0.077$ & 0.075 & $0.0040(0.0010-0.0214)$ & $0.0101(0.0030-0.1328)$ & 0.016 \\
\hline
\end{tabular}

Table 3. The different proportions of the key gut microbiota in each subgroup divided by levels of proteinuria and hematuria.

\begin{tabular}{|c|c|c|c|c|c|c|c|c|}
\hline \multicolumn{2}{|l|}{ Parameters } & g-Bacteroides & g-Blautia & g-Subdoligranulum & g-Prevotella-9 & g-Prevotella-7 & g-Bifidobacterium & $\begin{array}{c}\text { g-Escherichia } \\
\text {-Shigella }\end{array}$ \\
\hline \multirow{2}{*}{ Gd-IgA1 } & $\mathrm{r}$ & -0.146 & -0.185 & -0.102 & -0.002 & -0.356 & -0.266 & -0.046 \\
\hline & $\mathrm{p}$ & 0.328 & 0.214 & 0.496 & 0.990 & $0.014^{*}$ & 0.070 & 0.757 \\
\hline \multirow{2}{*}{ LBP } & $\mathrm{r}$ & -0.021 & -0.078 & -0.112 & -0.014 & -0.322 & -0.301 & -0.165 \\
\hline & $\mathrm{P}$ & 0.887 & 0.602 & 0.454 & 0.927 & $0.027^{*}$ & $0.040^{*}$ & 0.269 \\
\hline \multirow{2}{*}{ sCD14 } & $\mathrm{r}$ & -0.016 & -0.107 & -0.154 & 0.032 & -0.321 & -0.230 & -0.153 \\
\hline & $\mathrm{p}$ & 0.916 & 0.474 & 0.303 & 0.829 & $0.028^{*}$ & 0.119 & 0.306 \\
\hline \multirow{2}{*}{ ICAM-1 } & $\mathrm{r}$ & -0.031 & -0.052 & -0.070 & 0.028 & -0.311 & -0.249 & -0.194 \\
\hline & $\mathrm{p}$ & 0.839 & 0.731 & 0.639 & 0.851 & $0.033^{*}$ & 0.091 & 0.192 \\
\hline \multirow{2}{*}{ CRP } & $\mathrm{r}$ & -0.001 & -0.114 & -0.116 & -0.011 & -0.259 & -0.215 & -0.123 \\
\hline & $\mathrm{p}$ & 0.995 & 0.446 & 0.438 & 0.944 & 0.078 & 0.147 & 0.411 \\
\hline \multirow{2}{*}{ g-Escherichia-Shigella } & $\mathrm{r}$ & -0.124 & -0.125 & -0.091 & 0.191 & -0.305 & -0.116 & - \\
\hline & $\mathrm{p}$ & 0.406 & 0.403 & 0.544 & 0.200 & $0.037^{*}$ & 0.437 & - \\
\hline
\end{tabular}

Table 4. Spearman analysis of inflammatory indicators in serum and the genus proportion of fecal samples. Abbrevitions: Gd-IgA1, galactose deficiency of IgA1; LBP, Lipopolysaccharide binding protein; sCD14, soluble cluster of differentiation CD14; ICAM-1, intercellular adhesion molecule-1; CRP, C-reactive protein; A stands for $\mathrm{P}<0.05$.

\section{Figures}




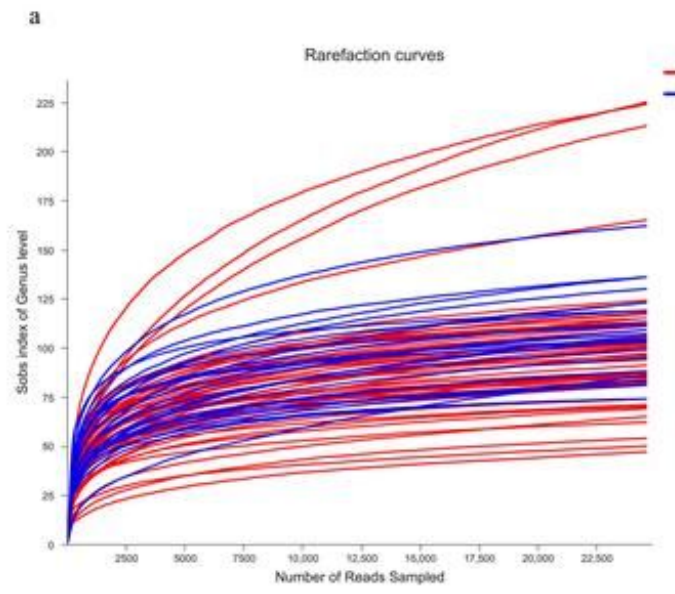

b 二

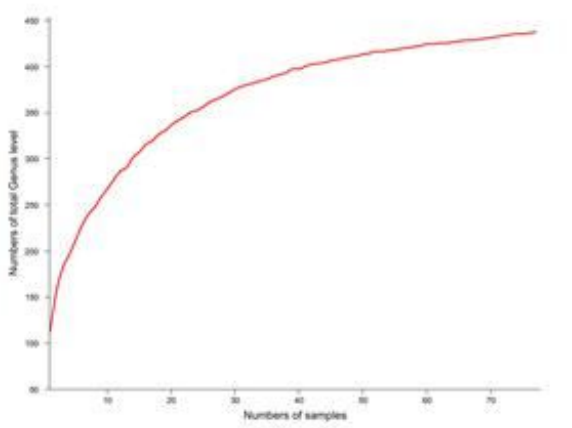

- All

Core analysis

- All

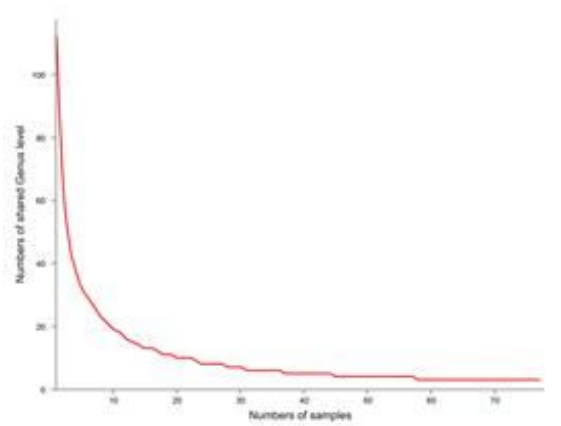

\section{Figure 1}

Rarefaction curves and pan/core genome analysis. (a) The rarefaction curves of IgAN patients and healthy controls (HCs) at the genus level. The sequencing depth was judged to be sufficient as the curve tended to be flat. The detection rate of the microbial community was almost flat, revealing a reasonable sequencing volume that could cover most species. (b) Pan-genome analysis. (c) Core-genome analysis. Pan and core analysis both describe the changes in core species members with increasing sample size. 
a
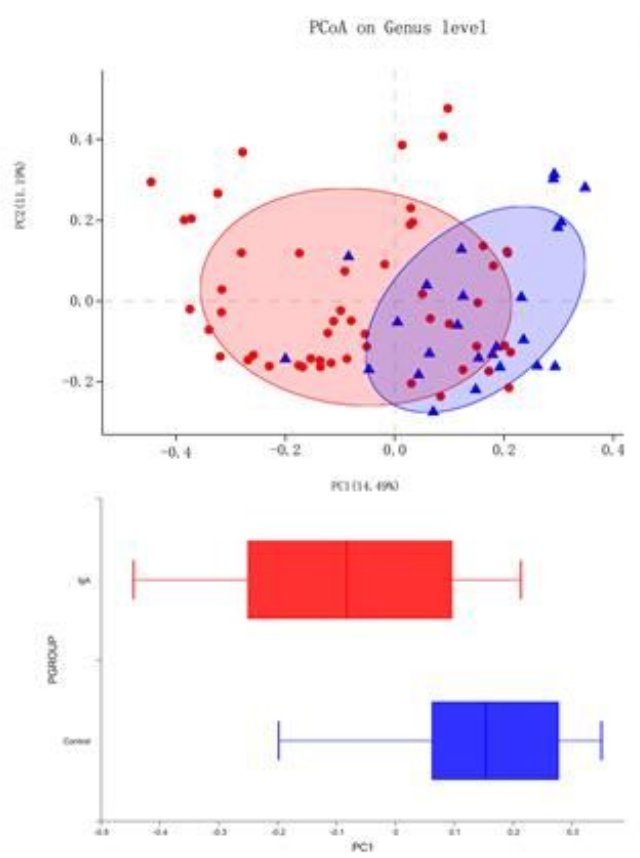

b
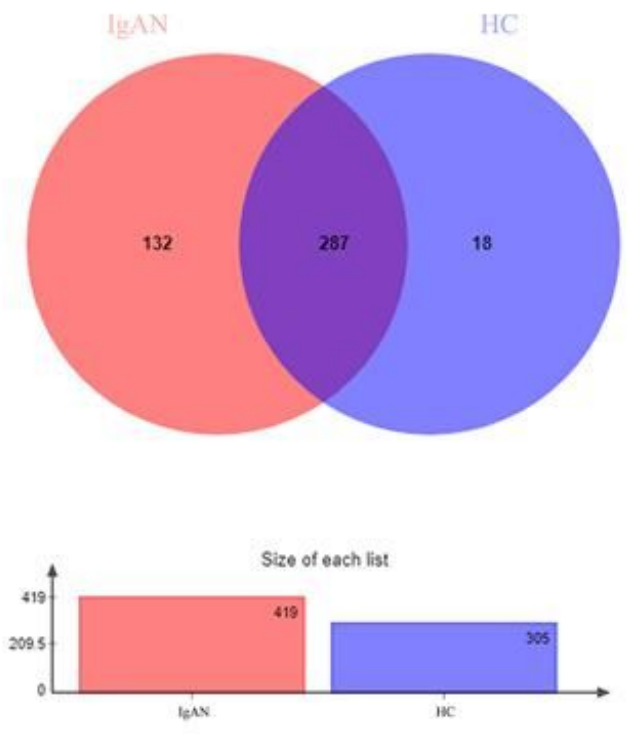

Number of elements: specific (1) or shared by 2, 3,... lists

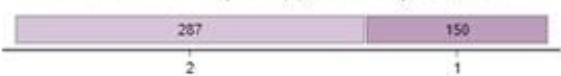

\section{Figure 2}

Principle Coordinate Analysis (PCoA) and Venn diagram of gut microbiota from IgAN patients and healthy controls (HCs) at the genus level. (a) PCoA. Triangles and circles represent different samples from the two groups. The structure and composition of the gut microbiota in IgAN patients were substantially different from those of HCs. (b) Venn diagram. There were 287 species shared between the two groups while 18 and 132 species were specific to the HCs and IgAN patients, respectively. 
a

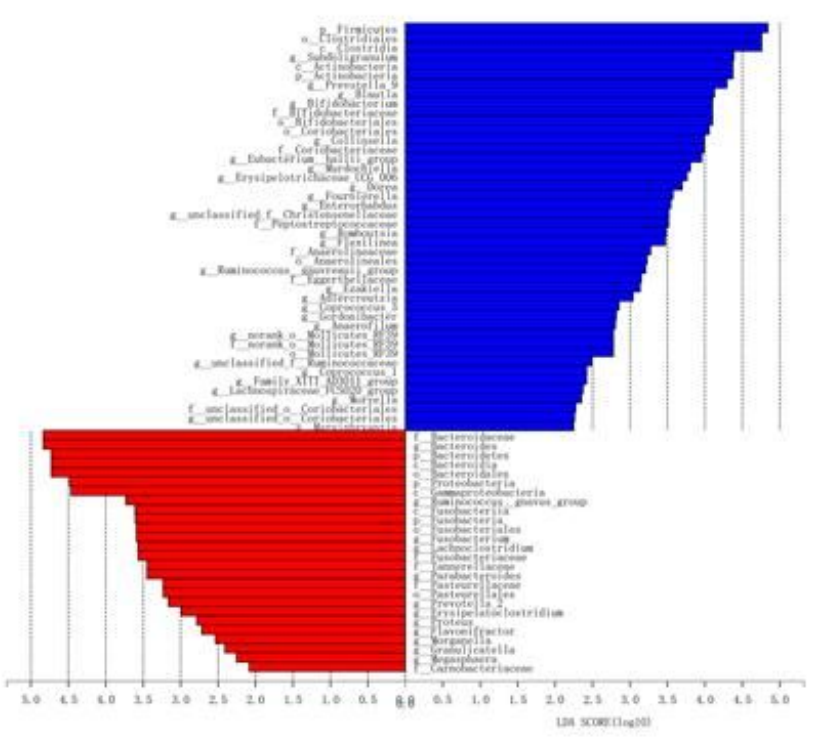

b

Cladogram

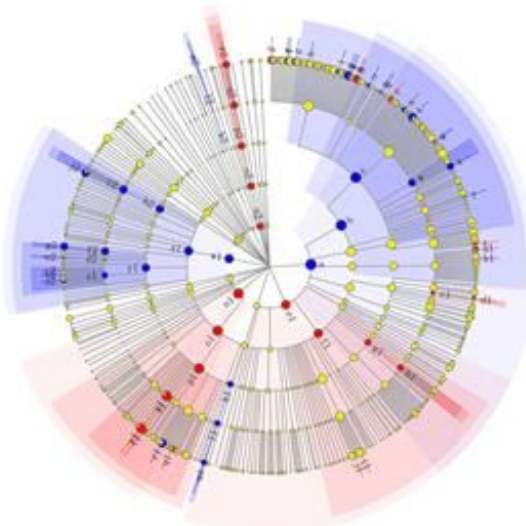

$\mathrm{HC}$

IgAN
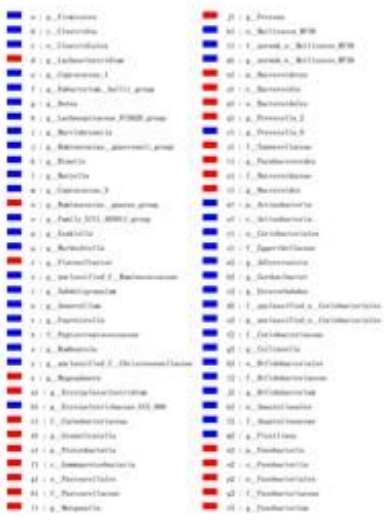

\section{Figure 3}

Gut microbiota differences between IgAN patients and healthy controls (HCs) were identified using a logarithmic linear discriminant analysis (LDA) effect size (LefSe) threshold >2.0. (a) Histogram of the LDA scores for the differential abundance of each species between IgAN patients and HCs. (b) Cladograms of bacterial lineages with obviously different between two groups at various levels from phylum to genus. Twenty-six bacterial taxa were significantly enriched in IgAN patients (red) while 45 bacterial taxa were significantly enriched in HCs (blue). 
a

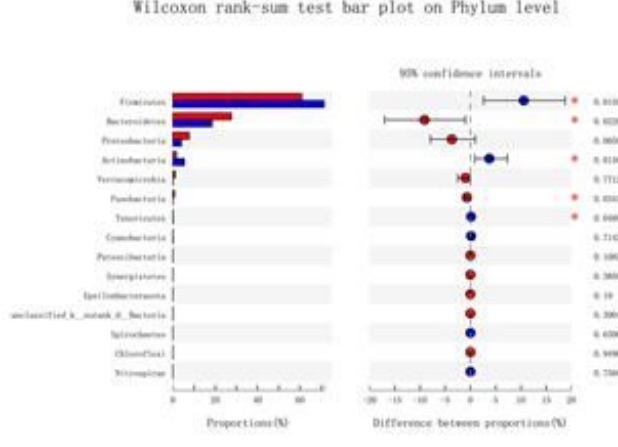

b

Vilcowon rank-suan test bar plot on Genus level

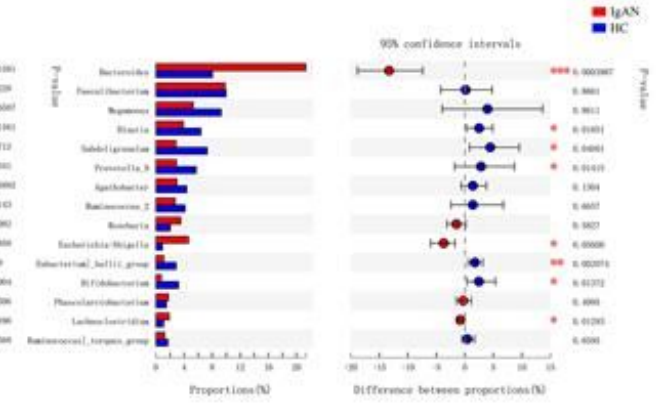

$\mathrm{c}$

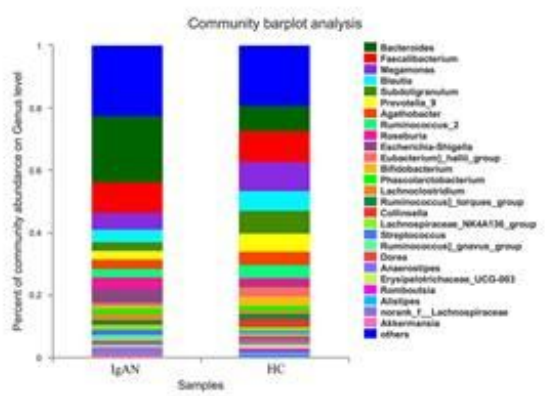

\section{Figure 4}

Relative abundance of the gut microbiota and Wilcoxon rank-sum test analysis between IgAN patients and healthy controls (HCs). (a) The relative abundance of gut microflora at the phylum level suggested that significant variation existed between the two groups. (b) Analysis of differences in the gut microbiota between the two groups at the genus level showed marked differences. (c) Community bar plot analysis showing the differential relative abundance of microflora between the two groups. 

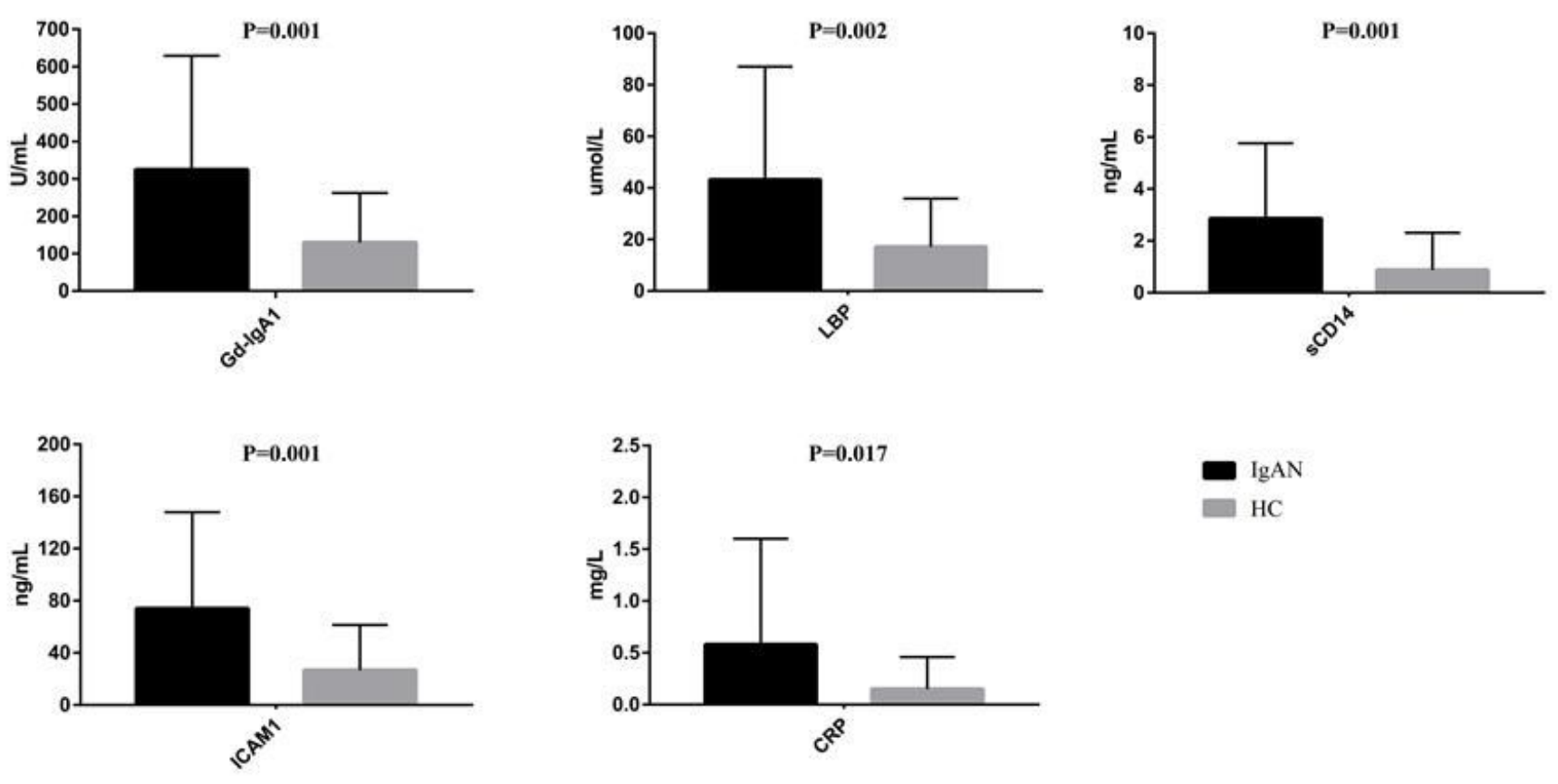

\section{Figure 5}

The relative serum levels of galactose-deficient IgA1 (Gd-lgA1), lipopolysaccharide binding protein (LBP), soluble cluster of differentiation 14 (SCD14), intercellular adhesion molecule 1 (ICAM-1), and C-reactive protein (CRP) measured by ELISA. 